\title{
The Cyclosporine-A Treatment does not have Harmful Effect on the Linear Growth of Pediatric Patients with Steroid-dependent and Steroid-resistant Nephrotic Syndrome
}

\author{
Sang Soo Lee, M.D. ${ }^{1}$ \\ Ji Hoon Kim, M.D. ${ }^{1}$ \\ Chung Ho Kim, M.D., Ph.D. ${ }^{2}$ \\ Byoung-Soo Cho, M.D., Ph.D. ${ }^{3}$ \\ Deog Yoon Kim, M.D., Ph.D. ${ }^{4}$ \\ II Ki Hong, M.D., Ph.D. ${ }^{4}$ \\ Jin-Soon Suh, M.D., Ph.D. ${ }^{1}$ \\ Department of Pediatrics' and \\ Nuclear Medicine ${ }^{2}$, Bucheon St. Mary's \\ Hospital, College of Medicine, The \\ Catholic University of Korea, Seoul, \\ Korea, Director of MIRAE-iNG Kidney \\ Center ${ }^{3}$, Seoul, Korea, Department of \\ Nuclear Medicine ${ }^{4}$, School of Medicine, \\ Kyung Hee University, Seoul, Korea \\ Corresponding author: \\ Jin-Soon Suh, M.D, Ph.D. \\ Department of Pediatrics, Bucheon St. \\ Mary's Hospital, College of Medicine, The \\ Catholic University of Korea, 327 Sosa-Ro, \\ Wonmi-Gu, Pucheon-Si, Gyeonggi-do, \\ 14647, Korea \\ Tel: +82-32-340-7047 \\ Fax: $+82-32-340-2673$ \\ E-mail: rebekahjs@hanmail.net \\ Received: 13 July 2016 \\ Revised: 27 September 2016 \\ Accepted: 10 October 2016 \\ This is an open-access article distributed \\ under the terms of the Creative Commons \\ Attribution Non-Commercial License (http:// \\ creativecommons.org/licenses/by-nc/4.0/) \\ which permits unrestricted non-commercial \\ use, distribution, and reproduction in any \\ medium, provided the original work is \\ properly cited.
}

Copyright (C) 2016 The Korean Society of Pediatric Nephrology
Purpose: This study was performed to evaluate the effects of cyclosporine-A (CsA) on linear growth in pediatric patients with steroid-dependent (SDNS) or resistant nephrotic syndrome (SRNS).

Methods: Thirty-five pediatric patients with SDNS or SRNS undergoing glucocorticoid (GC) and/or CsA treatment were retrospectively reviewed. Seventeen patients were treated with GC alone and 18 were treated with GC and CsA. The cumulative doses of GC and CsA were quantified ( $\mathrm{mg} / \mathrm{kg} / \mathrm{day}$ ). Linear growth during the follow-up period was defined as the difference in Z-score between the initial and final height according to the follow-up period ( $\Delta$ height $Z$ score/year). The associations between linear growth and clinical parameters were analyzed.

Results: The linear growth of patients in the two groups was not significantly different $(P=0.262)$. The $\Delta$ height $Z$ score/year did not show a significant correlation with the cumulative doses of $C S A$, but was negatively correlated with the cumulative dose of GC and positively correlated with the $Z$ score for height at the time of diagnosis.

Conclusion: In children with SDNS or SRNS undergoing GC therapy, added CSA treatment may not have harmful effects on linear growth.

Key words: Cyclosporine A, Glucocorticoid, Children, Nephrotic syndrome, Growth

\section{Introduction}

Idiopathic nephrotic syndrome, one of the most common nephropathies occurring in childhood, is characterized by edema, nephrotic-range proteinuria, hypoalbuminemia, and hyperlipidemia. Glucocorticoid (GC) therapy is the mainstay of treatment for childhood nephrotic syndrome. Although it has much improved the prognosis of these patients, the long-term use of GC may have several adverse effects, including growth retardation and bone loss, especially in growing children ${ }^{1,2)}$. Therefore, nephrotic patients who relapse frequently or are steroid resistant require steroid-sparing immunosuppressants, including cyclosporine A (CsA) $)^{3}$. Compared with the harmful effects of GC on growth and metabolic bone disease in nephrotic patients, CsA is regarded as a safer drug for growing children ${ }^{4,5}$. However, its effects on growth 
in nephrotic children are largely unknown. Therefore, the present study was performed to evaluate the effects of CsA on linear growth in pediatric patients with steroid-dependent nephrotic syndrome (SDNS) and steroid-resistant nephrotic syndrome (SRNS).

\section{Materials and methods}

\section{Subjects}

Data from 35 pediatric patients with SDNS or SRNS who received GC treatment with or without CsA at Kyung Hee University Hospital and Bucheon St. Mary's Hospital of the Catholic University of Korea between March 2003 and December 2014 were reviewed retrospectively. SDNS was defined when patients had two consecutive relapses during corticosteroid therapy, or within 14 days of ceasing therapy, and SRNS was defined when children with nephrotic syndrome did not achieve remission after 8 weeks treatment of daily corticosteroid ${ }^{6,7}$. Subjects who were treated with other immunosuppressants, such as cyclophosphamide and mycophenolate mofetil, or had other diseases that could affect growth, including genetic syndromes and bone diseases, were excluded. To exclude the effects of growth hormone (GH) treatment on the linear growth of children, in cases in which GH treatment was applied, data from the period prior to the initiation of this treatment were collected. The patients enrolled in this study were divided into two groups: those treated with GC alone $(\mathrm{n}=17)$ and those treated with $\mathrm{GC}$ and CsA ( $\mathrm{n}=18)$. The cumulative doses of GC and CsA received by each child during the follow-up period were quantified and reported as milligrams per kilogram per day. The institutional review boards of Kyung Hee University Hospital and Bucheon St. Mary's Hospital of the Catholic University of Korea approved this study.

\section{Measurement of linear growth}

The initial height, assessed at the time of diagnosis, the height at starting CsA treatment, and final height, assessed at the end of the follow-up period, were expressed as Z scores according to the standards of the 2007 Korea National Growth Charts8). Linear growth during the follow-up period was defined as the difference between the $\mathrm{Z}$ scores of the initial and final heights ( $\Delta$ height $Z$ score/year).

\section{Statistical analyses}

Data were presented as means \pm standard deviations (SD) for normally distributed variables and as medians and interquartile ranges for nonparametric variables. Differences between groups were analyzed using unpaired Student's ttests (for variables with parametric distributions) and the Mann-Whitney U-test (for variables with nonparametric distributions). Associations between linear growth and clinical parameters were analyzed using Spearman's correlation coefficient (r). Linear regression was used to evaluate the associations of the cumulative doses of GC and CsA with linear growth in two separate univariate models. Multivariate analyses were used to adjust for potential confounders, such as sex, age, duration of follow up, and initial height $Z$ score. Statistical analyses were performed using SPSS software (version 20.0; IBM Corporation, Armonk, NY, USA). Significance was defined as $P<0.05$.

\section{Results}

\section{Baseline characteristics}

All subjects in the GC group had SDNS. In the CsA group, 7 patients had SDNS and 11 had SRNS. The subjects in the CsA group were well matched with those in the GC group with regard to sex distribution, age at the time of diagnosis, age at the end of follow up, duration of follow up, height at the time of diagnosis, and cumulative dose of GC received during the observational period (Table 1).

\section{Effects of CsA and GC on the linear growth of children with SDNS and SRNS}

The linear growth of patients ( $\Delta$ height $\mathrm{Z}$ score/year) did not differ significantly between GC group and CsA group $(P=0.262$; Table 1$)$. Furthermore, the linear growth was not associated significantly with the cumulative CsA dose (Table 2). In contrast, the linear growth of patients was associated negatively with the cumulative dose of GC and positively with the $\mathrm{Z}$ score for height at the time of diagnosis in two independent univariate analyses. These significant associations remained evident in multivariate analyses after adjustment for other clinical parameters: sex, age at diagnosis, age at the end of follow up, duration of follow up, and cumulative dose of CsA (Table 2). In addition, in the 
Table 1. Clinical Characteristics and Growth Data of Children with Steroid-dependent and Steroid-resistant Nephrotic Syndrome

\begin{tabular}{|c|c|c|c|}
\hline & GC group $(n=17)$ & CsA group $(n=18)$ & $P$ value \\
\hline SDNS:SRNS & $17: 0$ & $7: 11$ & \\
\hline $\operatorname{Sex}(M: F)$ & $10: 7$ & $16: 2$ & 0.096 \\
\hline Age at diagnosis (years) & $5.87 \pm 2.15$ & $5.01 \pm 2.03$ & 0.229 \\
\hline Age at the end of follow up (years) & $8.40 \pm 1.90$ & $8.69 \pm 2.33$ & 0.690 \\
\hline Duration of follow-up period (months) & $36.2(22.2-45.23)$ & $41.7(24.25-62.08)$ & 0.373 \\
\hline Duration of administering GC (months) & $22.5(6.81-43.3)$ & $15.4(11.80-25.33)$ & 0.817 \\
\hline${ }^{11}$ Cumulative dose of GC (mg/kg/day) & $0.57(0.36-0.69)$ & $0.42(0.27-0.53)$ & 0.121 \\
\hline Before administering CsA & & $2.34(0.64-3.64)$ & 0.006 \\
\hline Since administering CsA & & $0.21(0.07-0.45)$ & \\
\hline Duration of administering CsA (months) & & $13.9(7.6-40.7)$ & \\
\hline Cumulative dose of CsA (mg/kg/day) & & $0.85(0.54-1.43)$ & \\
\hline${ }^{2)}$ Height at diagnosis (Z score) & $0.36 \pm 1.56$ & $-0.18 \pm 0.93$ & 0.223 \\
\hline${ }^{3)}$ Height at the end of follow up (Z score) & $-0.26 \pm 1.31$ & $-0.19 \pm 0.91$ & 0.853 \\
\hline${ }^{4)} \Delta$ height Z score/year & $-0.06(-0.42-0.11)$ & $-0.01(-0.08-0.06)$ & 0.262 \\
\hline${ }^{5)}$ Height at starting CsA (Z score) & & $-0.28 \pm 0.93$ & \\
\hline${ }^{6)} \Delta$ height Z score/year before administering CsA & & $0(-0.01-0)$ & 0.001 \\
\hline${ }^{77} \Delta$ height Z score/year since administering CsA & & $0.21(0.07-0.45)$ & \\
\hline
\end{tabular}

Abbreviations: GC group: patients treated with glucocorticoid only; CSA group: patients treated with glucocorticoid and cyclosporine A; SDNS: steroiddependent nephrotic syndrome; SRNS: steroid-resistant nephrotic syndrome; 4) $\Delta$ height Z score: difference between Z scores 2) and 3); 6) $\Delta$ height Z score: difference between Z scores 2) and 5); 7) $\Delta$ height Z score: difference between Z scores 3) and 5).

Table 2. Regression Analyses of Linear Growth in Children with Nephrotic Syndrome

\begin{tabular}{|c|c|c|c|c|c|c|c|c|}
\hline & \multicolumn{4}{|c|}{ Univariate } & \multicolumn{4}{|c|}{ Multivariate } \\
\hline & Standard $\beta$ & $P$ value & $\mathrm{R}$ & $\mathrm{Cl}(95 \%)$ & Standard $\beta$ & $P$ value & $\mathrm{R}$ & $\mathrm{Cl}(95 \%)$ \\
\hline Cumulative dose of steroids & -0.540 & 0.001 & 0.348 & $-0.351 \sim 0.008$ & -0.543 & $<0.001$ & 0.746 & $-0.709 \sim 0.790$ \\
\hline Cumulative dose of cyclosporine & -0.004 & 0.983 & 0.475 & $-0.181 \sim-0.002$ & -0.068 & 0.615 & & $-0.317 \sim 0.062$ \\
\hline Sex & & & & & -0.255 & 0.074 & & $-0.096 \sim 0.625$ \\
\hline Age at diagnosis & & & & & -0.045 & 0.749 & & $-0.266 \sim 0.132$ \\
\hline Age at the end of follow up & & & & & -0.026 & 0.846 & & $-0.150 \sim 0.259$ \\
\hline Duration of follow-up period & & & & & -0.006 & 0.964 & & $-0.019 \sim 0.018$ \\
\hline Z score of height at the time of diagnosis & & & & & -0.410 & 0.003 & & $-0.186 \sim 0.035$ \\
\hline
\end{tabular}

Abbreviation: $\mathrm{Cl}$, confidence interval.

CsA group, the cumulative dose of GC before the administration of CsA was higher than the cumulative dose of GC since the administration of CsA $(P=0.006)$. And the $\Delta$ height $Z$ score/year since the administration of CsA was higher than the linear growth before the administration of CsA $(P=0.001)$ (Table 1$)$.

\section{Discussion}

In the present study, the effect of GC combined with CsA treatment on the linear growth of nephrotic patients did not differ from that of treatment with GC alone. Results of two previous studies suggested that CsA therapy had no harmful effect, and indeed had a positive effect, on linear growth $^{4,9)}$. Kano et al. reported that the height $\mathrm{Z}$ scores of 8 boys with SDNS had increased by $0.4 \mathrm{SD}$ at 1 year after the start of CsA therapy and by $0.7 \mathrm{SD}$ at 2 years after the start of CsA therapy ${ }^{9)}$. Moreover, the degree of increase in height $\mathrm{Z}$ score since the start of CsA therapy was greater than that since the cessation of prednisolone, which was supported by increases in serum alkaline phosphatase and urinary $\mathrm{GH}$ concentrations after the start of CsA therapy ${ }^{9}$. Ishikura et al. reported that the linear growth of patients with relapsing nephrotic syndrome was maintained or slightly improved during 2 years of follow up after 2 years of CsA ad- 
ministration, despite the administration of prednisolone ${ }^{4)}$. Results of other studies of the effects of immunosuppressants on the growth of nephrotic patients have suggested that growth retardation is caused mainly by long-term use of GC, and not by other immunosuppressants ${ }^{1,10,11)}$. However, heterogeneous types and doses of immunosuppressants including CsA and mycophenolate mofetil were used in these studies. Therefore, estimation of the effects of the drugs, including CsA, on growth may be difficult. In the present study, we included prepubertal subjects undergoing GC treatment with and without CsA, but not with other immunosuppressants. Therefore, the effects of immunosuppressants other than CsA on growth are excluded. We suggest that it is the strength of this study. However, because the GC dose was decreased since the administration of CsA, the own effects of CsA on the growth of nephrotic patients remain unclear.

The observed effects of GC on growth were in accordance with those reported previously ${ }^{11,12)}$. The cumulative dose of GC and height at the time of diagnosis were correlated with the linear growth of patients. Other researchers have proposed various mechanisms for the growth retardation caused by GC. Long-term GC use may cause dysregulation of several growth-regulating processes, such as the GHinsulin-like growth factor-1 (IGF-1)-insulin-like growth factor binding protein (IGFBP) axis and the secretion of $\mathrm{GH}^{13)}$. Steroids may affect the growth plate directly and decrease chondrocyte proliferation ${ }^{14)}$. Alternatively, nephrotic syndrome itself can cause dysregulation of the GH-IGF-1IGFBP axis and loss of growth-related proteins in urine, resulting in growth retardation ${ }^{15)}$. However, few studies have investigated the mechanism underlying the effects of CsA on growth and related processes. As CsA is an important and commonly used steroid-sparing agent for nephrotic syndrome in children, further investigations of its effects on growth and the underlying mechanism(s) are warranted.

In conclusion, in children with SDNS or SRNS undergoing steroid therapy, the addition of CsA to the treatment regimen may not have a harmful effect on linear growth. However, the steroid dose was associated negatively with linear growth.

\section{Conflicts of Interest}

No potential conflict of interest relevant to this article was reported.

\section{References}

1. Motoyama $\mathrm{O}$, litaka K. Final height in children with steroid-sensitive nephrotic syndrome. Pediatr Int 2007;49:623-5.

2. Gulati S, Sharma RK, Gulati K, Singh U, Srivastava A. Longitudinal follow-up of bone mineral density in children with nephrotic syndrome and the role of calcium and vitamin D supplements. Nephrol Dial Transplant 2005;20:1598-603.

3. Ishikura K, Ikeda M, Hattori S, Yoshikawa N, Sasaki S, lijima K, et al. Effective and safe treatment with cyclosporine in nephrotic children: a prospective, randomized multicenter trial. Kidney Int 2008;73:1167-73.

4. Ishikura K, Yoshikawa N, Nakazato H, Sasaki S, lijima K, Nakanishi $\mathrm{K}$, et al. Two-year follow-up of a prospective clinical trial of cyclosporine for frequently relapsing nephrotic syndrome in children. Clin J Am Soc Nephrol 2012;7:1576-83.

5. Freundlich M. Bone mineral content and mineral metabolism during cyclosporine treatment of nephrotic Syndrome. J Pediatr 2006;149:383-9.

6. Lombel RM, Gipson DS, Hodson EM, Kidney Disease: Improving Global O. Treatment of steroid-sensitive nephrotic syndrome: new guidelines from KDIGO. Pediatr Nephrol 2013;28:415-26.

7. Lombel RM, Hodson EM, Gipson DS, Kidney Disease: Improving Global O. Treatment of steroid-resistant nephrotic syndrome in children: new guidelines from KDIGO. Pediatr Nephrol 2013;28: 409-14.

8. Seo JY, Cho YG, Kang JH, Hur YI, Park HA, Kim KW, et al. New diagnostic criteria for obesity and overweight in Korean children and adolescents using 2007 Korean National Growth Charts. Obes Res Clin Pract 2013;7:e182-9.

9. Kano K, Nishikura K, Hoshi M, Yamada Y, Arisaka O. Linear growth of low-dose cyclosporin A therapy in children with steroid-dependent nephrotic syndrome. Nephron 2001;87:293-4.

10. Ishikura K, Yoshikawa N, Nakazato H, Sasaki S, Nakanishi K, Matsuyama T, et al. Morbidity in children with frequently relapsing nephrosis: 10-year follow-up of a randomized controlled trial. Pediatr Nephrol 2015;30:459-68.

11. Leroy V, Baudouin V, Alberti C, Guest G, Niaudet P, Loirat C, et al. Growth in boys with idiopathic nephrotic syndrome on longterm cyclosporin and steroid treatment. Pediatr Nephrol 2009; 24:2393-400.

12. Ribeiro D, Zawadynski S, Pittet LF, Chevalley T, Girardin E, Parvex P. Effect of glucocorticoids on growth and bone mineral density in children with nephrotic syndrome. Eur J Pediatr 2015;174:911-7. 
www.chikd.org

13. Olney RC. Mechanisms of impaired growth: effect of steroids on bone and cartilage. Horm Res 2009;72 Suppl 1:30-5.

14. Klaus G, Jux C, Fernandez P, Rodriguez J, Himmele R, Mehls O. Suppression of growth plate chondrocyte proliferation by cor- ticosteroids. Pediatr Nephrol 2000;14:612-5.

15. Dong F, Ren J. Insulin-like growth factors (IGFs) and IGF-binding proteins in nephrotic syndrome children on glucocorticoid. Pharmacol Res 2003;48:319-23. 